\title{
DNASE1L3 Deficiency, New Phenotypes and Evidence for a Transient Type I Interferon Signaling
}

\section{Maud Tusseau}

The International Center of Research in Infectiology, Lyon University

\section{Ema Lovšin}

University Children's Hospital University Medical Center Ljubljana

Charlotte Samaille

Nephrologie pediatrique, Hôpital Jeanne de Flandre

\section{Remi Pescarmona}

The International Center of Research in Infectiology, Lyon University

\section{Maria Cristina Maggio}

University of Palermo

\section{Velma Selmanovic}

Clinical center University of Sarajevo

\section{Marusa Debeljak}

University Children's Hospital University Medical Center Ljubljana

\section{Angelique Dachy}

Nephrologie pediatrique, Hôpital Jeanne de Flandre

\section{Alexandre Janin}

Biochemistry and Molecular Biology Department, Lyon University Hospital

\section{Louis Januel}

NeuroMyoGene Institute, Lyon 1 University

\section{Anne-Laure Mathieu}

The International Center of Research in Infectiology, Lyon University

\section{Jean Baptiste Gibier}

Univ. Lille, UMR9020-U1277 - CANTHER - Cancer Heterogeneity Plasticity and Resistance to Therapies

\section{Emilie Chopin}

Hospices Civils de Lyon, Centre de Biotechnologie Cellulaire et Biothèque,

\section{Isabelle Rouvet}

Hospices Civils de Lyon, Centre de Biotechnologie Cellulaire et Biothèque,

\section{David Goncalves}

Immunology Department, Hospices Civils de Lyon, Centre Hospitalier Lyon Sud

\section{Nicole Fabien}

Immunology Department, Hospices Civils de Lyon, Centre Hospitalier Lyon Sud

\section{Gillian Rice}


edicine and Health, University of Manchester

\section{Audrey Labalme}

Genetics Department, Lyon University Hospital

\section{Paolo Romagnani}

University of Milano

\section{Gaetan Lesca}

Genetics Department, Lyon University Hospital

\section{Thierry Walzer}

The International Center of Research in Infectiology, Lyon University

\section{Sebastien Viel}

The International Center of Research in Infectiology, Lyon University

\section{Magali Perret}

The International Center of Research in Infectiology, Lyon University

\section{Yannick Crow}

Université de Paris

\section{Tadej Avčin}

University Children's Hospital University Medical Center Ljubljana

\section{Rolando Cimaz}

University of Milano

\section{Alexandre Belot ( $\nabla$ alexandre.belot@chu-lyon.fr )}

The International Center of Research in Infectiology, Lyon University https://orcid.org/0000-0003-49025332

\section{Research Article}

Keywords: DNASE1L3, systemic lupus erythematosus, apoptosis, ANCA, nucleic acid

Posted Date: May 24th, 2021

DOI: https://doi.org/10.21203/rs.3.rs-502604/v1

License: (c) (1) This work is licensed under a Creative Commons Attribution 4.0 International License. Read Full License 


\section{DNASE1L3 deficiency, new phenotypes and evidence for a transient type I interferon signaling.}

Authors: M Tusseau ${ }^{1,2}$, E Lovsin $^{3}$, C Samaille ${ }^{4}$, R Pescarmona ${ }^{1,5}$, AL Mathieu ${ }^{1}$, MC Maggio $^{6}$, V Selmanović ${ }^{7}$, M Debeljak ${ }^{3}$, A Dachy ${ }^{4}$, A Janin ${ }^{8,9}$, L Januel $^{9}$, JB Gibier ${ }^{10}$, E Chopin ${ }^{11}$, I Rouvet ${ }^{11}$, D Goncalves ${ }^{12}$, N Fabien ${ }^{12}$,

GI Rice ${ }^{13}$, G Lesca $^{2}$, A Labalme $^{2}$, P Romagnani ${ }^{14}$, T Walzer ${ }^{1}$, S Viel ${ }^{1,12}$, M Perret $^{1,12}$, YJ Crow ${ }^{15,16}$, T Avčin ${ }^{3}$, R Cimaz ${ }^{14}$, A Belot ${ }^{1,17,18}$

1: The International Center of Research in Infectiology, Lyon University, INSERM U1111, CNRS UMR 5308, ENS, UCBL, Lyon, France

2: Genetics Department, Lyon University Hospital, Lyon, France.

3 : University Children's Hospital University Medical Center Ljubljana, Ljubljana, Slovenia.

4: Nephrologie pediatrique, Hôpital Jeanne de Flandre, CHU Lille, France.

5: Immunology Laboratory, Hospices Civils de Lyon, Lyon Sud Hospital, Pierre Benite, France

6: University Department Pro.Sa.M.I. "G. D'Alessandro," University of Palermo, Palermo, Italy.

7: Clinical center University of Sarajevo, Bosnia and Herzegovina.

8: Cardiogenetics Laboratory, Biochemistry and Molecular Biology Department, Lyon University Hospital, Lyon, France

9: NeuroMyoGene Institute, Lyon 1 University, CNRS UMR 5510, INSERM U1217, Lyon, France.

10: Univ. Lille, UMR9020-U1277 - CANTHER - Cancer Heterogeneity Plasticity and Resistance to Therapies, F-59000 Lille, France.

11: Hospices Civils de Lyon, Centre de Biotechnologie Cellulaire et Biothèque, Bron, France.

12: Immunology Department, Hospices Civils de Lyon, Centre Hospitalier Lyon Sud, Lyon, France.

13: Division of Evolution and Genomic Sciences, School of Biological Sciences, Faculty of Biology, Medicine and Health, University of Manchester, Manchester Academic Health Science Centre, Manchester, UK.

14: ASST G. Pini, Milano Italy and Department of Clinical Sciences and Community Health, University of Milano, Italy.

15: Laboratory of Neurogenetics and Neuroinflammation, Institut Imagine, Université de Paris, Paris, France.

16:Centre for Genomic and Experimental Medicine, MRC Institute of Genetics and Molecular Medicine, University of Edinburgh, Edinburgh, United Kingdom.

17: National Referee Centre for Rheumatic and Autoimmune Diseases in Children, RAISE, Paris and Lyon, France.

18: Pediatric Nephrology, Rheumatology, Dermatology Department, Hôpital Femme Mère Enfant, Hospices Civils de Lyon, Bron, France.

Corresponding Author : Pr Alexandre BELOT

Email adress : alexandre.belot@chu-lyon.fr 


\section{Abstract:}

Deoxyribonuclease 1 like 3 (DNASE1L3) is a secreted enzyme that has been shown to digest the extracellular chromatin derived from apoptotic bodies, and DNASE1L3 pathogenic variants have been associated to a lupus phenotype. It is unclear whether interferon signaling is sustained in DNASE1L3 deficiency in humans. Here we report four new patients carrying biallelic DNASE1L3 pathogenic variations, including two previously unreported mutations. Disease in one patient was characterized by lupus nephritis and skin lesions, while two others exhibited hypocomplementemic urticarial vasculitis syndrome. The fourth patient presented with earlyonset inflammatory bowel disease. To explore whether or not the interferon cascade was strongly and sustainably induced, Interferon stimulated genes (ISGs) expression was assessed for each patient. Contrary to canonical type-I interferonopathies, we noticed a transient increase of ISGs in blood, which reverted to normal with disease remission. Reviewing previous reports, DNASE1L3-related disease appears to carry a significant risk of lupus nephritis and a poor outcome together with the presence of anti-neutrophil cytoplasmic antibodies (ANCA). DNASE1L3 deficiency may share the pathogenesis with C1q deficiency by affecting efferocytosis, and this report suggests that interferon production is not directly driven by DNASE1L3 pathogenic variants.

Keywords: DNASE1L3; systemic lupus erythematosus; apoptosis; ANCA; nucleic acid 


\section{Introduction}

Systemic lupus erythematosus (SLE) is an autoimmune disease characterized by the production of antibodies against nucleic acids and an upregulation of type-I interferon in the majority of patients. The etiology of the disease is unexplained in most cases, but is thought to involve both genetic and environmental factors. Over the last 5 years, we and others have taken advantage of Next Generation Sequencing (NGS) technology to explore the Mendelian contribution to juvenile-onset SLE (jSLE), predicted to be enriched for monogenic forms of the disease with 7\% of jSLE associated with inborn errors of immunity[1-7]. In 2011, AlMayouf et al. described 17 patients from six families with a diagnosis of SLE who were all homozygous for a frameshift mutation in DNASE1L3:c.643delT p.(Trp215Glyfs*2), suggestive of an Arabian founder mutation[8]. An absence of gene transcript was consistent with a nonsense-mediated decay and loss-offunction. Of note, these patients presented with severe disease including lupus nephritis and positive ANCA antibodies.

An additional phenotype was reported by Özçakar et al. in six cases from two distinct families demonstrating hypocomplementemic urticarial vasculitis syndrome (HUVS)[9]. Whole exome sequencing identified two deletions: a homozygous 2-pb deletion c.290_291delCA, p.(Thr97Ilefs*2) (reported as c.289_290delAC in the paper) and a homozygous deletion c.320+4_320+7delAGTA in each of the two families. In addition, the first deletion resulted in nonsense mediated mRNA decay, whilst the 4 bp deletion mutant led to skipping of exon 3. Then, Carbonella et al. reported another family with atypical lupus carrying the c.643delT frameshift mutation[10]. Finally, Batu et al. and Bruschi et al. described two other cases with jSLE carrying the c.290_291delAC variant[11,12].

Here, we report on four patients with pathogenic variations in DNASE1L3, including 2 previously undescribed causal variants, and expand the phenotype from SLE to vasculitis with gut involvement. We also review previous reports highlighting the spectrum of DNASE1L3 deficiency. 


\section{Methods}

Molecular analysis: Identification of disease-causing variants was based on NGS sequencing in 3 out of 4 patients, including three custom-designed gene panels. For patient 2, whose clinical and biological presentation strongly suggested a DNASE1L3 deficiency, coding regions of the DNASE1L3 gene were directly sequenced by Sanger sequencing (See supplemental data).

Numbering of DNASE1L3 variants is according to the complementary DNA sequence RefSeq NM_004944. Type I interferon signature: Whole blood was collected into PAXgene tubes, total RNA was extracted using a PreAnalytix RNA isolation kit and RNA concentration was assessed using a spectrophotometer (FLUOstar Omega, Labtech). Quantitative reverse transcription polymerase chain reaction (RT-qPCR) analysis was performed using the TaqMan Universal PCR Master Mix (Applied Biosystems), and complementary DNA (cDNA) derived from 40 ng total RNA. Using TaqMan probes for IFI27 (Hs01086370_m1), IFI44L (Hs00199115_m1), IFIT1 (Hs00356631_g1), ISG15 (Hs00192713_m1), RSAD2 (Hs01057264_m1), and SIGLEC1 (Hs00988063_m1), the relative abundance of each target transcript was normalized to the expression level of HPRT1 (Hs03929096_g1) and 18S (Hs999999001_s1), and assessed with the Applied Biosystems StepOne Software v2.1 and DataAssist Software v.3.01. For each of the 6 probes, individual data were expressed relative to a single calibrator. RQ (relative quantification) is equal to $2^{-\Delta \Delta \mathrm{Ct}}$ i.e. the normalized fold change relative to the control data. The median fold change of the 6 genes compared to the median of 29 previously collected healthy controls was used to create an interferon score for each individual. An abnormal interferon score is defined as greater than +2 standard deviations above the mean of the control group i.e. 2.466 .

For some samples, mRNA quantification of the 6 ISG transcripts was performed using nanostring technology (Nanostring Technologies, Seattle, WA). Data standardization was obtained using the geometric mean of housekeeping genes count number. Of note, RT-qPCR and nanostring technology display similar analytical performance, as previously reported. The threshold was set at 2.3[13].

Simoa assay: The Simoa assay was used to measure plasma IFN- $\alpha 2$ protein according to the manufacturer's instructions. Serum IFN- $\alpha 2$ concentrations ( $\mathrm{fg} / \mathrm{mL}$ ) were determined from frozen samples with an HD-1 Analyzer and using a commercial kit (Quanterix, Lexington, MA). The threshold was set at $30 \mathrm{fg} / \mathrm{mL}[14]$.

Minigene splicing reporter assay: As the $\mathrm{c} .433 \mathrm{G}>\mathrm{A}$ missense variant observed in patient 2 was predicted to affect a splice site (using prediction methods like SpliceSiteFinder-like, MaxEntScan, GeneSplicer), its effect was studied with a Minigene splicing reported assay as previously described[15]. The sequence containing the exon of interest with around 200 flanking bases in intron 4 and 5 was amplified using the Phusion High-Fidelity DNA Polymerase (New England Biolabs, Ipswich, MA). We used DNA from the father as he was carrying the variant in the heterozygous state. Amplicons were inserted in the NdeI restriction site of the previously described pTB minigene vector using the In-fusion HD polymerase chain reaction (PCR) cloning kit (Takara Bio, Kusatsu, Japan). Transfection in HeLa cells was conducted with $1 \mu \mathrm{g}$ of plasmid using Fugene (Promega, Madison, WI, USA). Forty-eight hours after transfection, total RNA was extracted from the cells using RNAqueous-4PCR Total RNA Isolation Kit (ThermoFisherScientific) and then treated by DNase (DNA-free 
DNA Removal Kit, ThermoFisherScientific). RT-PCR was performed with Transcriptor High Fidelity cDNA Synthesis Kit (Roche Molecular Diagnostics, Indianapolis, IN, USA) using $250 \mathrm{ng}$ of total RNA and random primers. cDNA amplification was performed using vector-specific primers surrounding the cloning site and HotStar Taq Plus DNA polymerase (Qiagen, Hilden, Germany). The PCR products were resolved on a 2\% agarose gel and sequenced to identify splicing events. All transfection experiments were performed in triplicate. 


\section{Results}

We report on four unrelated patients carrying DNASE1L3 variants (Fig 1a). The main phenotypic characteristics are summarized in Table 1, and medical histories are described in detail in the Supplemental data.

Patient 1 description: The first patient was a female child of Albanian ethnicity with a history of recurrent fever associated with fatigue, polymorphous erythematosus skin rash and non-erosive arthritis of the hands and feet (Fig 1b). Biological investigations revealed a hemolytic anemia, and urine analysis revealed persistent proteinuria. Remission was obtained within 6 months under treatment (methylprednisolone, mycophenolate mofetil and hydroxychloroquine), and the girl is now in long-term remission. The type I interferon signature was increased at the first assessment (9.5) but have returned to basal levels after clinical remission.

We identified the heterozygous frameshift variant c.289_290delAC (p.Thr97Ilefs*2) already reported in the homozygous state[9,10]. In addition, a large deletion of exon 5 was recorded in our patient, and confirmed by TAQman qPCR. This deletion is not present in gnomAD_V2.1 database and has not been previously described at the homozygous state in patients. Sequencing of the parents determined that they were heterozygous for one of these two variants, thus confirming biallelic inheritance.

Patient 2 description: This young girl of Arab descent was referred with bilateral arthralgia associated with an atypical urticaria and, after a few months of evolution, lupus like glomerulonephritis (Fig 1b). She was treated with corticosteroids, mycophenolate mofetil, hydroxychloroquine and tacrolimus, leading to remission two months later (negative proteinuria and ANA (antinuclear antibodies). The type I interferon signature was positive at the first assessment (12.4) but stepped down to normal values when her clinical disease remitted (1.4).

Using Sanger sequencing, we identified a homozygous variant c.433G $>$ A in DNASE1L3, and both parents were heterozygous for this same variant. This variant has never been reported in the homozygous state in gnomAD_V3.1 database (four times in the heterozygous state), and its position corresponds to the last base of exon 4 , suggesting a splicing effect. Splicing prediction softwares predicted a reduced strength of the adjacent donor site (Fig 2b). Using a minigene assay, we confirmed an effect of this variant leading to exon 4 skipping, shifting the reading frame and terminating at position Ter19 (Fig 2c). This variant can thus be classified as pathogenic using ACMG criteria[16].

Patient 3 description: The third patient was an Italian girl presenting with recurrent fever episodes coupled with urticaria, arthralgia, myalgia and anemia. Non-steroidal anti-inflammatory drugs were effective in reducing pain but not on urticaria. She was then treated with corticosteroids, hydroxychloroquine and azathioprine. The type I interferon signature and IFN- $\alpha 2$ concentration were assessed whilst under NSAIDs and with active disease, and the result was positive ( 5 and $44.5 \mathrm{fg} / \mathrm{mL}$, respectively).

Targeted panel sequencing revealed compound heterozygous variants of the DNASEIL3 gene: the 2-bp deletion c.289_290delAC already reported by Özçakar et al.[9], and a second novel missense variant c.321- 
$1 \mathrm{G}>\mathrm{A}$, absent from the gnomAD_V3.1 database. As this substitution affects the canonical AG donor splice site, it can be classified as pathogenic (Fig 2b).

Patient 4 description: This patient is a boy whose symptoms started at the age of 1.,5 years with recurrent episodes of urticaria, vomiting, abdominal pain, mucus in the stools, poor weight gain and arthritis. Biopsy of a skin showed leukocytoclastic vasculitis and endoscopy revealed lymphocytic and eosinophilic infiltration in the small intestine and colona bowel inflammation. He had low level of $\mathrm{Clq}$ and highly positive anti-C1q antibodies and was diagnosed with hypocomplementemic urticarial vasculitis syndrome. Corticosteroids, and azathioprine, cyclophosphamide and later mycophenolate mofetil treatment lead to partial improvement but resulted in steroid dependency. Then, from the age of 5-6 years old, he presented marked systemic inflammation with febrile episodes that were improved by repeated courses of rituximab, but he did not achieve sustained disease remission. At age of 10.5 years he developed new type of skin involvement with bullous and hemorrhagic vasculitis, and serological signs of SLE with positive ANA, anti-dsDNA, anticardiolipin antibodies and lupus anticoagulants. Both the type I interferon signature and the IFN- $\alpha 2$ concentration were negative ( 0.7 and below the detection limit)

when the patient was in the state of low disease activity on remission at 11 years old and was negative. NGS based genetic analysis disclosed the previously described loss of function homozygous deletion c.653delT[8]. 


\section{Discussion}

We have recently shown that approximately $7 \%$ of jSLE are caused by monogenic inborn errors of immunity[1], mainly represented by innate immunity defects with complement and DNASE1L3 deficiencies. DNASE1L3 pathogenic variants were first identified as a cause of monogenic jSLE by Al Mayouf et al.[8]. In contrast, the patients reported by Özçakar et al. were described as suffering HUVS and the family reported by Carbonella et al. presented an atypical lupus phenotype with joint contractures[9,10].

Here we describe four further cases of DNASE1L3 deficiency. Pt 1 was previous reported in a large scale genetic analysis in 117 SLE patients[1]. The first patient displayed early-onset jSLE with systemic involvement including nephritis, and remission was obtained using a conventional immunosuppressive regimen. Patients 2 and 3 showed clinical features compatible with HUVS. We describe a new phenotype, with inflammatory bowel disease as the predominant clinical manifestation in patient 4 . Considering the 28 patients so-far described with variants in DNAESIL3 (summarised in Table 1), our literature review highlights that renal involvement is a major disease feature, being the cause of death in two patients. On the basis of clinical features only, it seems difficult to reliably differentiate monogenic forms of lupus from other lupus as already noticed in early-onset SLE[17]. Regarding serologic markers, however, ANCA were frequently positive and may represent a useful screening test. This subset of autoantibodies is typically identified in systemic vasculitis such as granulomatosis with polyangiitis and microscopic polyangiitis, and in inflammatory bowel disease, particularly ulcerative colitis, whilst being uncommon in sporadic lupus[18].

To date, monogenic lupus has been associated with three main mechanisms: (1) complement deficiencies (related to efferocytosis defect), (2) type I interferonopathies and (3) B cell apoptosis defects. DNASE1L3 and DNASE1 represent the two major serum nucleases, and are thought to limit autoimmunity by providing effective clearance of DNA from apoptotic bodies[19].

Recently, Sisirak et al. demonstrated that circulating DNASE1L3 is produced by dendritic cells and macrophages and is able to digest chromatin in apoptotic cell-derived microparticles. Accordingly, DNASE1L3-deficient mice and human patients demonstrated elevated cell free DNA levels in plasma, particularly in circulating microparticles, and murine and human autoantibody clones, as well as serum antibodies from human SLE patients, bound to DNASE1L3-sensitive chromatin on the surface of such microparticles[20]. DNASE1L3 deficiency in mice causes a rapid antibody response to dsDNA and chromatin independent of the STING signaling pathway, followed by an SLE-like disease[20].

Defective clearance of microparticles thus represents a potent trigger of autoimmunity. C1q plays a crucial role in the removal of apoptotic bodies, and deficiencies of early-components of the complement pathway are associated with an increased risk of SLE and type-I interferon production[21-23]. So-called efferocytosis represents a tightly regulated process by which phagocytes are recruited to sites of cell death, where they recognize and engulf dying cells to clear them without inducing inflammation[24]. Remarkably, DNASE1L3 deficient mice were rescued from their autoimmune phenotype by crossing them with MyD88deficient, but not STING-deficient, mice, suggesting that DNASE1L3 is not involved into the intracellular metabolism of nucleic acids, in contrast to the other nucleases like TREX1 and SAMHD1. 
The key role of DNASE1L3 in clearing microparticles associated cell free DNA has been emphasized by the recent discovery of neutralizing antibodies to DNASE1L3[25]. This new autoantibody mediated phenocopy of a genetic deficiency could be particularly relevant in sporadic SLE. In patients carrying these autoantibodies, the microparticles carry long DNA fragments that seems to activate more efficiency the innate immune system and enhance the interferon production by pDC. However, in comparison to type-I interferonopathies (i.e. due to abnormalities in the nucleic acid sensing or signaling), we found in DNASE1L3 patients a lower overexpression of interferon response that was resolved with remission of clinical disease. These observations thus support a distinct role of DNASE1L3 in IFN-I regulation. The potential association between type-I interferon and extracellular circulating DNA deserves further exploration.

\section{Declarations}

Ethics approvals: The study was approved by the Leeds (East) Research Ethics Committee (reference number 10/H1307/132) and the Medical Ethics Committee of Sud Est III (Lyon, France).

Consent to Participate: Parental written informed consent was provided for inclusion of clinical data and samples in the study.

Disclosure of Conflicts of Interest: The authors declare that they have no conflict of interest.

\section{Acknowledgements}

Y.J.C. acknowledges a state subsidy managed by the National Research Agency (France) under the "Investments for the Future" (ANR-10-IAHU-01), and the MSDAvenir fund (DEVO-DECODE Project).

\section{Authorship Contributions}

Not applicable 


\section{References}

1. Belot A, Rice GI, Omarjee SO, Rouchon Q, Smith EMD, Moreews M, et al. Contribution of rare and predicted pathogenic gene variants to childhood-onset lupus: a large, genetic panel analysis of British and French cohorts. Lancet Rheumatol [Internet]. 2020 [cited 2020 Jan 16];0. Available from: https://www.thelancet.com/journals/lanrhe/article/PIIS2665-9913(19)30142-0/abstract 2. Belot A, Cimaz R. Monogenic forms of systemic lupus erythematosus: new insights into SLE pathogenesis. Pediatr Rheumatol Online J. 2012;10:21.

3. Omarjee O, Picard C, Frachette C, Moreews M, Rieux-Laucat F, Soulas-Sprauel P, et al. Monogenic lupus: Dissecting heterogeneity. Autoimmun Rev. 2019;18:102361.

4. Crow YJ. Lupus: how much "complexity" is really (just) genetic heterogeneity? Arthritis Rheum. 2011;63:3661-4.

5. Belot A, Kasher P, Trotter E, Foray A, Debaud A, Rice G, et al. Protein kinase c $\delta$ deficiency causes mendelian systemic lupus erythematosus with B cell-defective apoptosis and hyperproliferation. Arthritis Rheum. 2013;65:2161-71.

6. Briggs TA, Rice GI, Daly S, Urquhart J, Gornall H, Bader-Meunier B, et al. Tartrate-resistant acid phosphatase deficiency causes a bone dysplasia with autoimmunity and a type I interferon expression signature. Nat Genet. 2011;43:127-31.

7. An J, Briggs TA, Dumax-Vorzet A, Alarcón-Riquelme ME, Belot A, Beresford M, et al. TartrateResistant Acid Phosphatase Deficiency in the Predisposition to Systemic Lupus Erythematosus. Arthritis Rheumatol Hoboken NJ. 2016;

8. Al-Mayouf SM, Sunker A, Abdwani R, Abrawi SA, Almurshedi F, Alhashmi N, et al. Loss-of-function variant in DNASE1L3 causes a familial form of systemic lupus erythematosus. Nat Genet. 2011;43:1186-8. 9. Özçakar ZB, Foster J, Diaz-Horta O, Kasapcopur O, Fan Y-S, Yalçınkaya F, et al. DNASE1L3 Mutations in Hypocomplementemic Urticarial Vasculitis Syndrome. Arthritis Rheum. 2013;65:2183-9.

10. Carbonella A, Mancano G, Gremese E, Alkuraya FS, Patel N, Gurrieri F, et al. An autosomal recessive DNASE1L3-related autoimmune disease with unusual clinical presentation mimicking systemic lupus erythematosus. Lupus. 2017;26:768-72.

11. Batu ED, Koşukcu C, Taşkıran E, Sahin S, Akman S, Sözeri B, et al. Whole Exome Sequencing in Earlyonset Systemic Lupus Erythematosus. J Rheumatol. 2018;45:1671-9.

12. Bruschi M, Bonanni A, Petretto A, Vaglio A, Pratesi F, Santucci L, et al. Neutrophil Extracellular Traps

(NETs) profiles in patients with incident SLE and lupus nephritis. J Rheumatol. 2019;

13. Pescarmona R, Belot A, Villard M, Besson L, Lopez J, Mosnier I, et al. Comparison of RT-qPCR and Nanostring in the measurement of blood interferon response for the diagnosis of type I interferonopathies. Cytokine. 2019;113:446-52.

14. Trouillet-Assant S, Viel S, Ouziel A, Boisselier L, Rebaud P, Basmaci R, et al. Type I Interferon in Children with Viral or Bacterial Infections. Clin Chem. 2020;66:802-8.

15. Millat G, Lafont E, Nony S, Rouvet I, Bozon D. Functional characterization of putative novel splicing mutations in the cardiomyopathy-causing genes. DNA Cell Biol. 2015;34:489-96.

16. Richards S, Aziz N, Bale S, Bick D, Das S, Gastier-Foster J, et al. Standards and guidelines for the interpretation of sequence variants: a joint consensus recommendation of the American College of Medical Genetics and Genomics and the Association for Molecular Pathology. Genet Med Off J Am Coll Med Genet. 2015;17:405-24.

17. Weill O, Decramer S, Malcus C, Kassai B, Rouvet I, Ginhoux T, et al. Familial and syndromic lupus share the same phenotype as other early-onset forms of lupus. Jt Bone Spine Rev Rhum. 2016;

18. Galeazzi M, Morozzi G, Sebastiani GD, Bellisai F, Marcolongo R, Cervera R, et al. Anti-neutrophil cytoplasmic antibodies in 566 European patients with systemic lupus erythematosus: prevalence, clinical associations and correlation with other autoantibodies. European Concerted Action on the Immunogenetics of SLE. Clin Exp Rheumatol. 1998;16:541-6.

19. Napirei M, Ludwig S, Mezrhab J, Klöckl T, Mannherz HG. Murine serum nucleases - contrasting effects of plasmin and heparin on the activities of DNase1 and DNase1-like 3 (DNase113). FEBS J. 2009;276:105973.

20. Sisirak V, Sally B, D’Agati V, Martinez-Ortiz W, Özçakar ZB, David J, et al. Digestion of Chromatin in Apoptotic Cell Microparticles Prevents Autoimmunity. Cell. 2016;166:88-101.

21. Crow YJ. Type I interferonopathies: Mendelian type I interferon up-regulation. Curr Opin Immunol. 2015;32:7-12.

22. Troedson C, Wong M, Dalby-Payne J, Wilson M, Dexter M, Rice G, et al. Systemic lupus erythematosus 
due to $\mathrm{C} 1 \mathrm{q}$ deficiency with progressive encephalopathy, intracranial calcification and acquired moyamoya cerebral vasculopathy. Lupus. 2013;22:639-43.

23. Santer DM, Hall BE, George TC, Tangsombatvisit S, Liu CL, Arkwright PD, et al. C1q deficiency leads to the defective suppression of IFN-alpha in response to nucleoprotein containing immune complexes. $\mathrm{J}$ Immunol Baltim Md 1950. 2010;185:4738-49.

24. Green DR, Oguin TH, Martinez J. The clearance of dying cells: table for two. Cell Death Differ. 2016;23:915-26.

25. Hartl J, Serpas L, Wang Y, Rashidfarrokhi A, Perez OA, Sally B, et al. Autoantibody-mediated impairment of DNASE1L3 activity in sporadic systemic lupus erythematosus. J Exp Med.

2021;218:e20201138. 
Figure legends

Fig 1 a Pedigrees of the 4 reported cases. Squares: males; circles: females; black: affected variant carriers; WT: wild-type DNASE1L3 allele. b Clinical manifestations of patients with DNASE1L3 pathogenic variants. Polymorphous erythematous skin rash of the hand of patient 1 and urticaria of patient 2. c Renal biopsy images of patient 2. Left part of the figure: (Magnification x200, Masson's trichrome stain) Fibrocellular crescent occupies Bowman's space with focal rupture of capillary wall and fibrinoid necrosis (not shown here). The glomerular tufts uninvolved by crescent formation reveal significant mesangial and endocapillary hypercellularity. Immunofluorescence revealed subendothelial deposits with a "full-house" pattern. Right part of the figure: From top to bottom, left to right, immunofluorescence with anti IgA, IgG, C3 and C1q. d Results of type I IFN signature scores of the 4 reported cases (DNASE1L3), in comparison with healthy controls (HC) and 3 patients with type 1 interferonopathies (TYPE 1 IFN) (two patients with SAMHD1 pathogenic variants and one patient with TMEM173 pathogenic variant). Each color represents a different patient (Pt 1: orange; $\mathrm{Pt}$ 2: red; Pt 3: yellow; Pt 4: brown). Circles notifie patients that were untreated (taking into account immunosuppressive and targeted therapies) and triangles treated patients. The dotted line indicates the positivity threshold

Fig 2 a Annotation of all reported pathogenic variants in human DNASE1L3 gene in reference to transcript NM_04944.3. Exons are represented by grey boxes. All variants are associated with a truncating effect. Novel variants are in orange. b Characteristics the novel DNASE1L3 variants. Both are predicted to affect splicing and lead to exon 4 skipping, as one is located on the canonical AG splice site (patient 3 ) and the other involves the last base of exon 4 next to the donor splice site (patient 2). C Molecular characterization of the splicing effect of c.433 G>A variant carried by patient 2 using a Minigene assay. The agarose gel electrophoresis shows RT-PCR (reverse transcription polymerase chain reaction) transcripts results obtained after transfection of HeLa cells with wild-type (WT) and mutated constructs. The WT construct shows a normal splicing with the exon 4 . The mutated Minigene is associated with a complete effect of exon 4 skipping 


\begin{tabular}{|c|c|c|c|c|c|c|c|c|c|c|}
\hline Cases & & Variant & Sex & Disease & $\begin{array}{l}\text { Age at } \\
\text { onset } \\
\text { (years) }\end{array}$ & $\begin{array}{l}\text { Lupus } \\
\text { nephritis }\end{array}$ & Other features & Autoantibodies & Outcome & Ref \\
\hline \multirow{2}{*}{ Family 1} & Pt1 & \multirow{2}{*}{$\begin{array}{l}\text { c.643delT } \\
\text { p.Trp215Glyfs*2 }\end{array}$} & $\mathrm{F}$ & jSLE & 2 & - & NA & ANA, anti-dsDNA, ANCA & NA & \multirow[t]{9}{*}{ [8] } \\
\hline & $\mathrm{Pt} 3$ & & $\mathrm{M}$ & jSLE & 5 & + & NA & ANA, anti-dsDNA, ANCA & NA & \\
\hline \multirow{4}{*}{ Family 2} & Pt4 & \multirow{4}{*}{$\begin{array}{l}\text { c.643delT } \\
\text { p.Trp215Glyfs*2 }\end{array}$} & $\mathrm{F}$ & jSLE & 2,5 & + & NA & $\begin{array}{l}\text { ANA,anti-dsDNA, ANCA, } \\
\text { anti-cardiolipin }\end{array}$ & NA & \\
\hline & $\mathrm{Pt} 5$ & & $\mathrm{M}$ & jSLE & 4,5 & + & NA & $\begin{array}{l}\text { ANA, anti-dsDNA, ANCA, } \\
\text { anti-cardiolipin }\end{array}$ & NA & \\
\hline & Pt6 & & $\mathrm{F}$ & jSLE & 6 & + & NA & $\begin{array}{l}\text { ANA, anti-dsDNA, ANCA, } \\
\text { anti-cardiolipin }\end{array}$ & NA & \\
\hline & Pt7 & & $\mathrm{M}$ & jSLE & 4,5 & + & NA & $\begin{array}{l}\text { ANA, anti-dsDNA, ANCA, } \\
\text { anti-cardiolipin }\end{array}$ & NA & \\
\hline \multirow{2}{*}{ Family 3} & Pt8 & \multirow{2}{*}{$\begin{array}{l}\text { c.643delT } \\
\text { p.Trp215Glyfs*2 }\end{array}$} & M & jSLE & 3 & + & NA & ANA, anti-dsDNA, ANCA, & NA & \\
\hline & Pt9 & & $\mathrm{M}$ & jSLE & 2 & + & NA & ANA, anti-dsDNA, ANCA & $\mathrm{NA}$ & \\
\hline Family 4 & Pt10 & $\begin{array}{l}\text { c.643delT } \\
\text { p.Trp215Glyfs*2 }\end{array}$ & $\mathrm{F}$ & jSLE & 4,5 & - & NA & ANA, anti-dsDNA, ANCA, & NA & \\
\hline \multirow{3}{*}{ Family 5} & Pt13 & \multirow{3}{*}{ c.643delT } & $\mathrm{M}$ & jSLE & 5 & + & NA & ANA, anti-dsDNA, ANCA, & NA & \multirow{5}{*}{ [8] } \\
\hline & Pt14 & & $\mathrm{M}$ & jSLE & 12 & + & NA & ANA, anti-dsDNA, ANCA, & NA & \\
\hline & Pt15 & & $\mathrm{M}$ & jSLE & 11 & - & NA & $\begin{array}{l}\text { ANA, anti-dsDNA, ANCA, } \\
\text { anti-cardiolipin }\end{array}$ & NA & \\
\hline \multirow{2}{*}{ Family 6} & Pt16 & \multirow{2}{*}{ c.643delT } & $\mathrm{M}$ & jSLE & 6 & - & NA & ANA, anti-dsDNA, ANCA, & NA & \\
\hline & Pt17 & & $\mathrm{M}$ & jSLE & 11 & - & NA & ANA, anti-dsDNA, ANCA, & NA & \\
\hline \multirow{3}{*}{ Family 7} & Pt18 & \multirow{3}{*}{ c.289_290delAC } & $\mathrm{F}$ & HUVS & 6 & + & ADP & ANCA & Death & \multirow[t]{3}{*}{ [9] } \\
\hline & Pt19 & & $\mathrm{F}$ & $\begin{array}{l}\text { HUVS + } \\
\text { jSLE }\end{array}$ & 2 & + & $\begin{array}{l}\text { Uveitis, } \\
\text { Arthritis/arthralgia, } \\
\text { ADP }\end{array}$ & $\begin{array}{l}\text { ANA, anti-dsDNA, ANCA, } \\
\text { Anti-C1q }\end{array}$ & $\begin{array}{l}\text { jSLE developped } \\
\text { at 5,5yo }\end{array}$ & \\
\hline & Pt20 & & $\mathrm{F}$ & HUVS & 2 & - & $\begin{array}{l}\text { Arthritis/arthralgia } \\
\text { ADP, uveitis }\end{array}$ & $\begin{array}{l}\text { ANA, anti-dsDNA, ANCA, } \\
\text { Anti-C1q }\end{array}$ & Active HUVS & \\
\hline Family 8 & Pt21 & $\begin{array}{l}\text { c. } 320+4 \_320+7 d \\
\text { elAGTA }\end{array}$ & $\mathrm{F}$ & $\begin{array}{l}\text { HUVS + } \\
\text { jSLE }\end{array}$ & 6 & + & $\begin{array}{l}\text { Arthritis/arthralgia, } \\
\text { ADP }\end{array}$ & $\begin{array}{l}\text { ANA, anti-dsDNA, (ANCA } \\
\text { not done) }\end{array}$ & Remission & [9] \\
\hline
\end{tabular}




\begin{tabular}{|c|c|c|c|c|c|c|c|c|c|c|}
\hline \multirow{4}{*}{ Family 9} & Pt23 & \multirow{4}{*}{ c.289_290delAC } & M & $\begin{array}{l}\text { SLE + } \\
\text { HUVS }\end{array}$ & 17 & + & $\begin{array}{l}\text { Arthritis, anemia, } \\
\text { lung vasculitis }\end{array}$ & ANA, anti-dsDNA, pANCA & $\begin{array}{l}\text { Pulmonary } \\
\text { hypertension }\end{array}$ & \multirow{4}{*}[10]{} \\
\hline & Pt 24 & & $\mathrm{~F}$ & $\begin{array}{l}\text { Unknown } \\
\text { nephropathy }\end{array}$ & NA & + & & NA & $\begin{array}{l}\text { Kidney } \\
\text { transplantation }\end{array}$ & \\
\hline & Pt 25 & & M & JIA & NA & - & & NA & $\begin{array}{l}\text { Death (car } \\
\text { accident) }\end{array}$ & \\
\hline & Pt 26 & & $\mathrm{~F}$ & $\begin{array}{l}\text { Rhumatoid } \\
\text { arthritis }\end{array}$ & NA & - & & NA & NA & \\
\hline Family 10 & Pt 27 & c.289_290delAC & $\mathrm{F}$ & jSLE & 2 & + & $\begin{array}{l}\text { Arthritis/arthralgia, } \\
\text { scleritis, pulmonary } \\
\text { involvement }\end{array}$ & $\begin{array}{l}\text { ANA, anti-dsDNA, (ANCA } \\
\text { not done) }\end{array}$ & $\begin{array}{l}\text { Rare flares of } \\
\text { skin and eyes } \\
\text { manifestations } \\
\end{array}$ & [11] \\
\hline Family 11 & Pt 28 & c.289_290delAC & M & jSLE & 10 & + & NA & NA & $\begin{array}{l}\text { Kidney } \\
\text { transplantation }\end{array}$ & [12] \\
\hline $\begin{array}{l}\text { Family A } \\
\text { Patient } 1\end{array}$ & Pt 29 & $\begin{array}{l}\text { c.289_290delAC + } \\
\text { exon } 5 \text { deletion }\end{array}$ & $\mathrm{F}$ & jSLE & 4 & + & $\begin{array}{l}\text { Arthritis/arthralgia } \\
\text { ADP }\end{array}$ & ANA, anti-dsDNA & Remission & $\begin{array}{l}\text { Case } \\
\text { report }\end{array}$ \\
\hline $\begin{array}{l}\text { Family B } \\
\text { Patient } 2\end{array}$ & Pt 30 & c. $433 \mathrm{G}>\mathrm{T}$ & $\mathrm{F}$ & HUVS & 11 & + & Arthritis/arthralgia & ANA, anti-dsDNA, ANCA & Remission & $\begin{array}{l}\text { Case } \\
\text { report }\end{array}$ \\
\hline $\begin{array}{l}\text { Family C } \\
\text { Patient } 3\end{array}$ & Pt 31 & $\begin{array}{l}\text { c. } 289 \_290 \mathrm{delAC}+ \\
\text { c.321-1G>A }\end{array}$ & $\mathrm{F}$ & HUVS & 15 & - & $\begin{array}{l}\text { Arthritis, myalgia, } \\
\text { anemia }\end{array}$ & ANA, ANCA+/- & Remission & $\begin{array}{l}\text { Case } \\
\text { report }\end{array}$ \\
\hline $\begin{array}{l}\text { Family D } \\
\text { Patient } 4\end{array}$ & Pt 32 & c.643delT & $\mathrm{M}$ & HUVs & 1.5 & - & IBD, arthralgia & ANA, anti dsDNA, ANCA & Active IBD & $\begin{array}{l}\text { Case } \\
\text { report }\end{array}$ \\
\hline Total & 32 & - & $\begin{array}{l}\text { Sex } \\
\text { ratio: } \\
0.88\end{array}$ & HUVS, jSLE & $\begin{array}{l}\text { Mean: } \\
6 \text { yo }\end{array}$ & $21 / 32$ & - & ANCA : $23 / 24$ & Death: $2 / 9$ & - \\
\hline
\end{tabular}

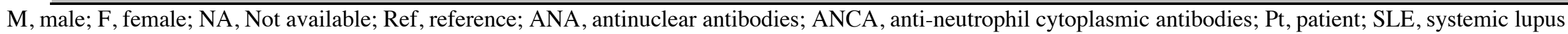
erythematosus; jSLE = juvenile SLE; HUVS, hypocomplementemic urticarial vasculitis syndrome; ADP, adenopathy; JIA, juvenile idiopathic arthritis; IBD, inflammatory bowel disease

Table 1 Characteristics of patients with DNASE1L3 deficiency 


\section{Figures}

a

Family $A$

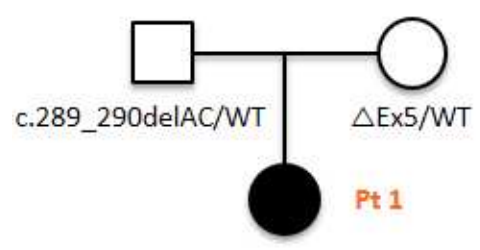

c.289_290delAC/ $\triangle$ Ex5
Family $B$

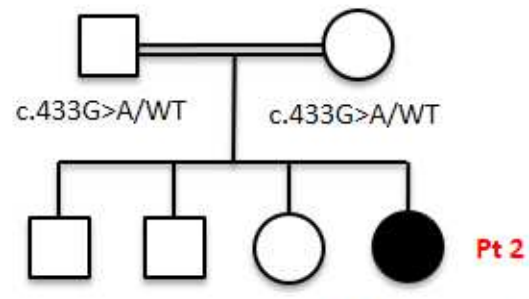

c. $433 \mathrm{G}>\mathrm{A} / \mathrm{c} .433 \mathrm{G}>\mathrm{A}$
Family $C$

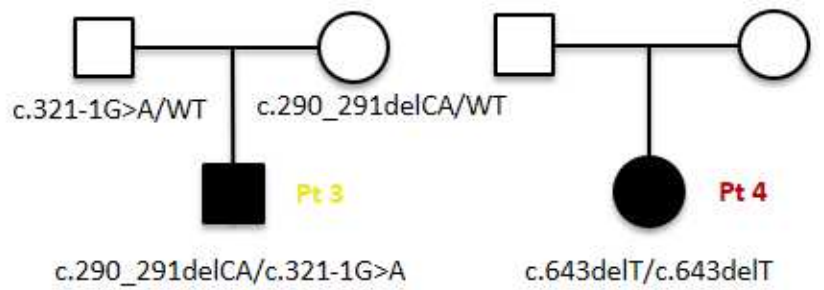

c. $290 \_291$ delCA/c.321-1G>A

c.643delT/c.643delT b

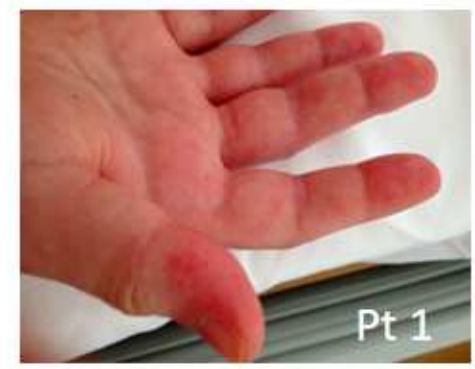

c

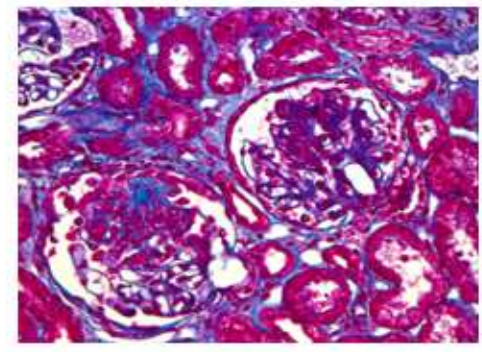

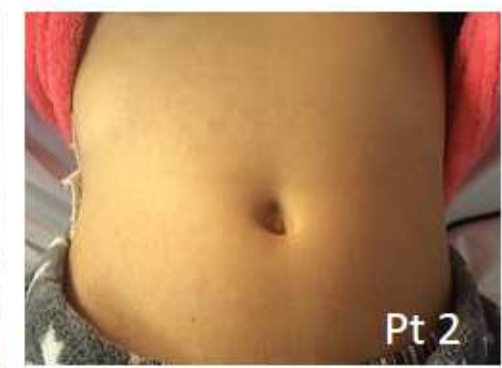

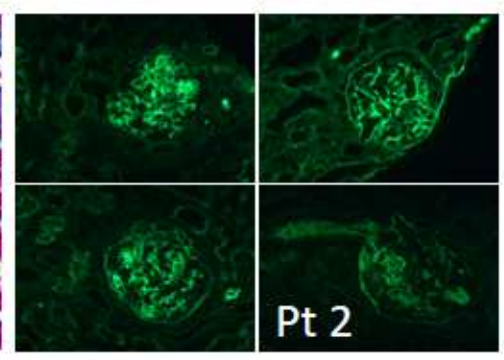

d

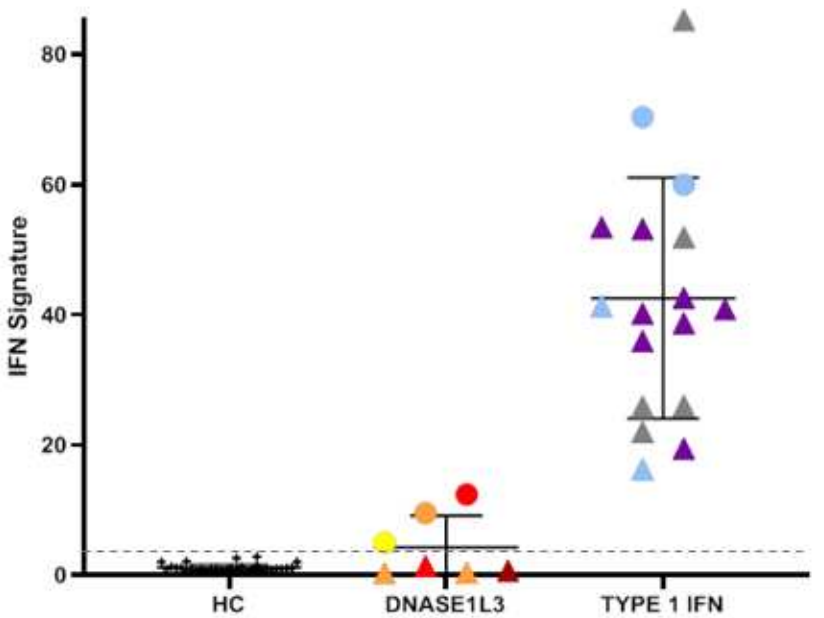

\section{Figure 1}

a Pedigrees of the 4 reported cases. Squares: males; circles: females; black: affected variant carriers; WT: wild-type DNASE1L3 allele. b Clinical manifestations of patients with DNASE1L3 pathogenic variants. Polymorphous erythematous skin rash of the hand of patient 1 and urticaria of patient 2. c Renal biopsy images of patient 2. Left part of the figure: Magnification x200, Masson's trichrome stain) Fibrocellular crescent occupies Bowman's space with focal rupture of capillary wall and fibrinoid necrosis (not shown here). The glomerular tufts uninvolved by crescent formation reveal significant mesangial and endocapillary hypercellularity. Immunofluorescence revealed subendothelial deposits with a "full-house" pattern. Right part of the figure: From top to bottom, left to right, immunofluorescence with anti IgA, IgG, C3 and C1q. d Results of type I IFN signature scores of the 4 reported cases (DNASE1L3), in comparison with healthy controls (HC) and 3 patients with type 1 interferonopathies (TYPE 1 IFN) (two patients with SAMHD1 pathogenic variants and one patient with TMEM173 pathogenic variant). Each color represents a different patient (Pt 1: orange; Pt 2: red; Pt 3: yellow; Pt 4: brown). Circles notifie patients that were untreated (taking into account immunosuppressive and targeted therapies) and triangles treated patients. The dotted line indicates the positivity threshold 


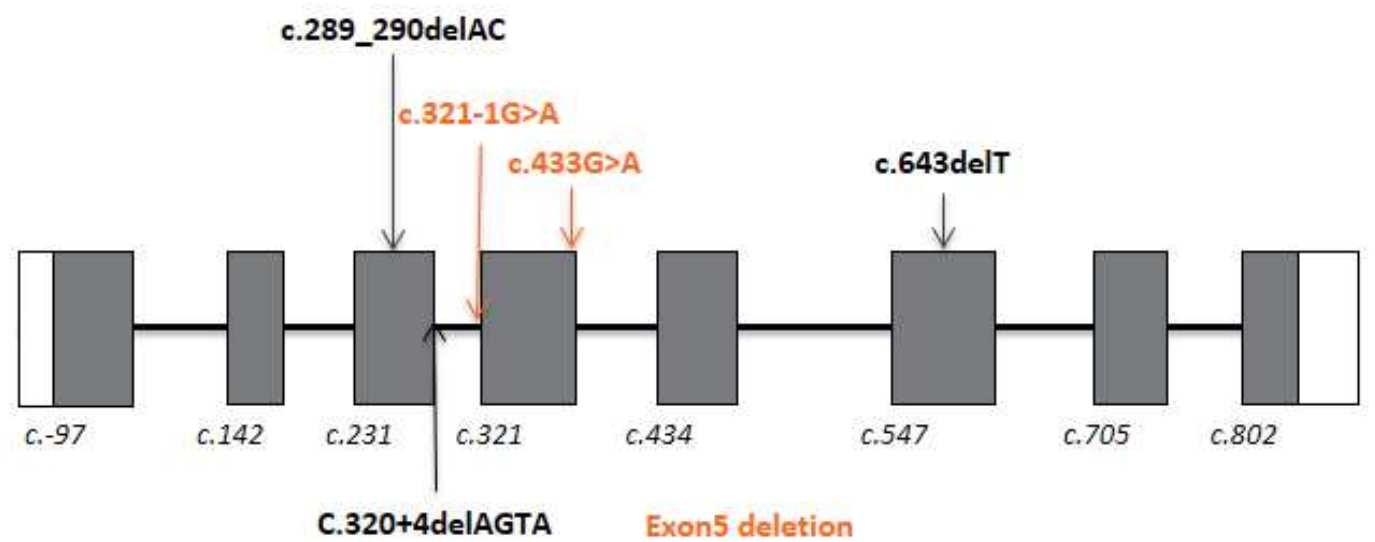

b

C

\begin{tabular}{|c|c|c|c|c|}
\hline Patient & Variant (HGVS) & $\begin{array}{c}\text { CADD } \\
\text { PHRED v1.6 }\end{array}$ & Predicted Effect & $\begin{array}{l}\text { Allele Frequency } \\
\text { (EnomAD V3.1.1) }\end{array}$ \\
\hline 2 & NM_004944.3:c.433G $>A$ & 33 & Exon 4 skipping & $\begin{array}{l}0.00001991 \\
\text { (heterozygous) }\end{array}$ \\
\hline 3 & NM_004944.3:c.321-1G>A & 33 & Exon 4 skipping & - \\
\hline
\end{tabular}

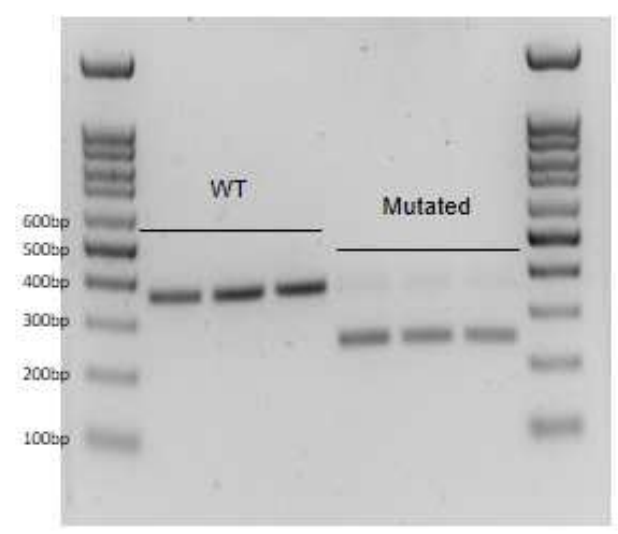

Figure 2

a Annotation of all reported pathogenic variants in human DNASE1L3 gene in reference to transcript NM_04944.3. Exons are represented by grey boxes. All variants are associated with a truncating effect. Novel variants are in orange. $b$ Characteristics the novel DNASE1L3 variants. Both are predicted to affect splicing and lead to exon 4 skipping, as one is located on the canonical AG splice site (patient 3 ) and the other involves the last base of exon 4 next to the donor splice site (patient 2). C Molecular characterization of the splicing effect of $c .433 \mathrm{G}>\mathrm{A}$ variant carried by patient 2 using a Minigene assay. The agarose gel electrophoresis shows RT-PCR (reverse transcription polymerase chain reaction) transcripts results obtained after transfection of HeLa cells with wild-type (WT) and mutated constructs. The WT construct shows a normal splicing with the exon 4. The mutated Minigene is associated with a complete effect of exon 4 skipping

\section{Supplementary Files}

This is a list of supplementary files associated with this preprint. Click to download.

- Supplementaldata.pdf 